\title{
Weak Wecken's theorem for periodic points in dimension 3
}

\author{
by \\ Jerzy Jezierski (Warszawa)
}

\begin{abstract}
We prove that a self-map $f: M \rightarrow M$ of a compact PL-manifold of dimension $\geq 3$ is homotopic to a map with no periodic points of period $n$ iff the Nielsen numbers $N\left(f^{k}\right)$ for $k$ dividing $n$ all vanish. This generalizes the result from [Je] to dimension 3 .
\end{abstract}

1. Introduction. We consider a self-map $f: X \rightarrow X$ of a topological space and its set of periodic points $\operatorname{Fix}\left(f^{n}\right)=\left\{x \in X ; f^{n}(x)=x\right\}$ for a fixed $n \in \mathbb{N}$. In 1982 Boju Jiang [Ji1] introduced a Nielsen type homotopy invariant $N F_{n}(f)$, which is a lower bound for the cardinality of the set of periodic points: \# $\operatorname{Fix}\left(f^{n}\right) \geq N F_{n}(f)$. Benjamin Halpern then conjectured that this number is the best such homotopy invariant (for self-maps of compact manifolds of dimension $\geq 5$ ), i.e. every self-map $f: M \rightarrow M$ of such a manifold is homotopic to a map $g$ satisfying \# Fix $\left(g^{n}\right)=N F_{n}(f)$ (Theorem 4.14 in Section III of [Ji1]). In [Je] we proved

THEOREM 1.1. Any self-map $f$ of a compact PL-manifold of dimension $\geq 4$ is homotopic to a map $g$ without periodic points of period $n\left(i . e . g^{n}(x)\right.$ $\neq x)$ iff the Nielsen number $N\left(f^{k}\right)$ is 0 for any divisor $k$ of $n$.

This answers positively the Halpern Conjecture under the assumption that $N F_{n}(f)=0$ since the last equality is equivalent to $N\left(f^{k}\right)=0$ for all $k$ dividing $n$.

In this paper we extend this result to manifolds of dimension 3. The main idea of this modification is "reparametrization of the path" (see the proof of Lemma 3.8). This allows us to minimize (in the homotopy sense) some inverse images and then to construct homotopies with no new periodic points. This in turn reduces the problem to a local one: see Theorem 3.1. We also hope that the present approach will give an easier proof of this result also in all dimensions $>3$.

Key words and phrases: fixed point, periodic point, Lefschetz number, Nielsen number. 
Let us add that in dimension 2 the Conjecture is not true since even in the case of fixed points the Nielsen number is usually much less than the least number of fixed points in the homotopy class of the given map (see $[\mathrm{K}]$ ).

I would like to thank the referee for a very careful reading which allowed me to remove numerous mistakes.

2. Preliminaries. In this section we introduce the basic notions of the Nielsen fixed point theory and we sketch the proof of (a weak form of) the classical Wecken theorem. Then we give an outline of the proof of its generalization to periodic points (Theorem 2.5), which is the main result of the present paper.

We consider a self-map $f: X \rightarrow X$ of a topological space $X$ and its fixed point set $\operatorname{Fix}(f)$. We define the Nielsen relation on $\operatorname{Fix}(f)$ by declaring $x \sim y$ iff there is a path $\omega:[-1,1] \rightarrow X$ such that $\omega(-1)=x, \omega(1)=y$ and the path $f \omega$ is fixed end point homotopic to $\omega$ (for some technical reasons we will use paths parametrized by the interval $[-1,1]$ rather than $[0,1]$ as usual). This relation splits $\operatorname{Fix}(f)$ into (a finite number of) mutually disjoint Nielsen classes. A Nielsen class $A$ is called essential if its fixed point index is nonzero: $\operatorname{ind}(f ; A) \neq 0$. The Nielsen number $N(f)$ is defined as the number of essential Nielsen classes. This number is a homotopy invariant $(f \sim g$ implies $N(f)=N(g))$ and is a lower bound of the cardinality of the fixed point set (\# $\operatorname{Fix}(g) \geq N(f)$ for all $g$ homotopic to $f$ ). Franz Wecken proved in 1942 [W] that the Nielsen number is the best such lower bound for all self-maps of manifolds of dimension $\geq 3$.

TheOREM 2.1 ([W]). Any self-map $f: M \rightarrow M$ of a compact topological manifold of dimension at least 3 is homotopic to a map having exactly $N(f)$ fixed points.

In particular for $N(f)=0$ we get

Corollary 2.2. A self-map $f: M \rightarrow M$ of a compact manifold of dimension at least 3 is homotopic to a map with no fixed points $\Leftrightarrow N(f)=0$.

Sketch of proof (cf. [Ji2]). $\Rightarrow$ is obvious since $N(f)$ does not exceed the number of fixed points of any map homotopic to $f$.

$\Leftarrow$ Let $f: M \rightarrow M$ satisfy $N(f)=0, \operatorname{dim} M \geq 3$. Then each Nielsen class $A \subset \operatorname{Fix}(f)$ is inessential, i.e. $\operatorname{ind}(f ; A)=0$. By a transversality lemma we may assume that $\operatorname{Fix}(f)$ is finite and $f$ is a linear homeomorphism near each fixed point, which implies $\operatorname{ind}(f ; x)= \pm 1$ for $x \in \operatorname{Fix}(f)$. Since $\operatorname{ind}(f ; A)=0, A$ splits into pairs $\left\{x_{1}, y_{1}, \ldots, x_{s}, y_{s}\right\}$ such that ind $\left(f ; x_{i}\right)+$ $\operatorname{ind}\left(f ; y_{i}\right)=0$. It remains to remove a pair $x_{i}, y_{i}$ by a homotopy which is constant in a neighbourhood of the remaining fixed points and which does not add any new fixed points. A path $\omega$ joining these two points and 
satisfying $f \omega \sim \omega$ can be chosen an arc. If we assume moreover that the image $f \omega$ is close to $\omega$ in the sense that there is a Euclidean neighbourhood containing both $\omega$ and $f \omega$ then the Hopf lemma and the assumption $\operatorname{ind}\left(f ; x_{i}\right)+\operatorname{ind}\left(f ; y_{i}\right)=0$ allow us to remove these two fixed points by a homotopy with carrier in a prescribed neighbourhood of $\omega$.

Thus the main problem in the general case ( $f \omega$ and $\omega$ distant) is to find a homotopy which does not change the fixed point set and after which the paths $\omega, f \omega$ are contained in a Euclidean neighbourhood. A homotopy making $f \omega$ close to $\omega$ exists by the definition of the Nielsen relation. The main difficulty is to avoid producing a new fixed point during this homotopy. If $\operatorname{dim} M \geq 3$ then the homotopy can be deformed to avoid the arc $\omega(0,1)$ since $M \backslash \omega \approx M \backslash *$ and the image of the homotopy of an arc is twodimensional.

We follow a similar scheme to prove the main theorem of the present paper. We will confine ourselves to PL-manifolds although in what follows only the proof of the referenced Theorem 2.4 uses PL arguments.

TheOREM 2.3. Any self-map $f: M \rightarrow M$ of a compact PL-manifold of dimension $\geq 3$ is homotopic to a map $g$ without periodic points of period $n$ (i.e. $\left.g^{n}(x) \neq x\right)$ iff $N\left(f^{k}\right)=0$ for any divisor $k$ of $n$.

Proof. $\Rightarrow$ is evident. The rest of the paper is the proof of $\Leftarrow$. We use induction with the respect to the divisors of the given $n \in \mathbb{N}$ : for every $k \mid n$ we show that

$f$ is homotopic to a map $g$ satisfying $g^{l}(x) \neq x$ for all $l \mid n, l \leq k$.

For $k=1$ this follows from the above Wecken theorem. Now we assume that it holds for all divisors of $n$ which are less than $k$. We will show how to remove the $k$-periodic points.

TheOREM 2.4 ([Je]). Let $M \subset \mathbb{R}^{N}$ be a compact $P L$-submanifold with metric induced by the Euclidean metric in $\mathbb{R}^{N}$. Fix $n \in \mathbb{N}$. Then any continuous map $f: M \rightarrow M$ is homotopic to a map $g$ such that $\operatorname{Fix}\left(g^{n}\right)$ is finite and $g$ is a PL-homeomorphism near any point $x \in \operatorname{Fix}\left(g^{n}\right)$. Moreover for any $\varepsilon>0$ we may choose $g$ to satisfy $d(f, g)<\varepsilon$.

By the above theorem we may assume that $\operatorname{Fix}\left(f^{n}\right)$ is finite and $f$ is a linear homeomorphism near each $x \in \operatorname{Fix}\left(f^{n}\right)$. In particular ind $\left(f^{k} ; x\right)= \pm 1$ at these points. Consider an orbit of Nielsen classes $A \subset \operatorname{Fix}\left(f^{k}\right)$. Since by the induction assumption $f^{l}(x) \neq x(l \mid n, l<n, x \in M)$, all orbits of points in $\operatorname{Fix}\left(f^{k}\right)$ have length $k$. Since $N\left(f^{k}\right)=0$, we have ind $\left(f^{k} ; A\right)=0$, hence $A$ splits into finitely many pairs of orbits $\left\{x_{1}, \ldots, x_{k}\right\},\left\{y_{1}, \ldots, y_{k}\right\}$ such that there is a path $\omega:[-1,1] \rightarrow M$ establishing the Nielsen relation between $x_{1}, y_{1}$ and $\operatorname{ind}\left(f^{k} ; x_{1}\right)+\operatorname{ind}\left(f^{k} ; y_{1}\right)=0$. 
Thus the induction step will be done once we prove that the orbits $\left\{x_{1}, \ldots, x_{k}\right\},\left\{y_{1}, \ldots, y_{k}\right\}$ can be removed by a homotopy which is constant in a neighbourhood of the other fixed points and which does not produce new fixed points. In other words, it remains to show the following

Theorem 2.5 (Cancelling Procedure). Let $f: M \rightarrow M$ be a map with $\operatorname{Fix}\left(f^{k}\right)$ finite $(\operatorname{dim} M \geq 3)$. Assume that

(1) $\left\{x_{0}, \ldots, x_{k-1}\right\},\left\{y_{0}, \ldots, y_{k-1}\right\}$ are disjoint orbits of length $k$ which are Nielsen related, i.e. there is a path $\omega:[-1,1] \rightarrow M$ from $f(-1)=$ $x_{0}$ to $f(1)=y_{0}$ such that $f^{k} \omega$ and $\omega$ are fixed end point homotopic,

(2) $f$ is a PL-homeomorphism near each point from $\left\{x_{0}, \ldots, x_{k-1}\right.$; $\left.y_{0}, \ldots, y_{k-1}\right\}$.

(3) $\operatorname{ind}\left(f^{k} ; x_{0}\right)+\operatorname{ind}\left(f^{k} ; y_{0}\right)=0$.

Then there is a homotopy $\left\{f_{t}\right\}$ starting from $f_{0}=f$ constant in a neighbourhood of $\operatorname{Fix}\left(f^{k}\right) \backslash\left\{x_{0}, \ldots, x_{k-1} ; y_{0}, \ldots, y_{k-1}\right\}$ and satisfying

$$
\operatorname{Fix}\left(f_{1}^{k}\right)=\operatorname{Fix}\left(f^{k}\right) \backslash\left\{x_{0}, \ldots, x_{k-1} ; y_{0}, \ldots, y_{k-1}\right\} .
$$

The rest of the paper is the proof of Theorem 2.5. We end this section by an outline of this proof.

Some technical lemmas allow us to make $\omega$ and its images $f \omega, \ldots, f^{k-1} \omega$ flat arcs and $f$ a homeomorphism in neighbourhoods of these arcs (more exactly in neighbourhoods of $f^{i} \omega[-1,0)$ and $f^{i} \omega(0,1]$ for $\left.i=0, \ldots, k-2\right)$.

If moreover it happens that $f^{k} \omega$ is close to $\omega$ then the use of a modified Hopf lemma allows us to remove the two orbits. Thus the main difficulty in the general case is to make $f^{k} \omega$ close to $\omega$ without adding new periodic points. For some technical reasons the image of such a homotopy should avoid the arc $f^{k-1} \omega$ and some of its inverse images. In [Je] we used some transversality arguments to reduce the dimension of these inverse images to 1 . Thus the assumption $\operatorname{dim} M \geq 4$ allowed us to deform the (two-dimensional) homotopy to avoid these (one-dimensional) inverse images. This is the only place in [Je] which does not work in dimension 3. In this paper we modify the last deformation so as not to produce new periodic points also in dimension 3 . The main idea of this modification is a reparametrization of the segment $\omega_{k-1}=f^{k-1} \omega$.

3. Proof of Theorem 3.1. The crucial step in the proof of Theorem 2.5 is the following theorem which makes the path $f^{k} \omega$ close to $\omega$. This step corresponds to the reduction to the Euclidean case in the proof of the classical Wecken theorem. Let $V \subset \mathbb{R}^{m-1} \times \mathbb{R}$. We set

$$
\begin{gathered}
V^{+}=\{(x, t) \in V ; t>0\}, \quad V^{-}=\{(x, t) \in V ; t<0\}, \\
V^{0}=\{(x, t) \in V ; t=0\} .
\end{gathered}
$$


Theorem 3.1. Under the assumptions of the Cancelling Procedure (Theorem 2.5) there exists a homotopy which does not change $\operatorname{Fix}\left(f^{k}\right)$, is constant in a neighbourhood of $\operatorname{Fix}\left(f^{k}\right)$, and after which the map $f$ satisfies:

(1) a path $\omega_{0}:[-1,1] \rightarrow M$ establishing the Nielsen relation between $x_{0}, y_{0} \in \operatorname{Fix}\left(f^{k}\right)$ is a PL-arc avoiding other periodic points,

(2) there exist mutually disjoint Euclidean neighbourhoods $V_{0}^{\prime}, \ldots, V_{k-1}^{\prime}$ such that $f^{i} \omega(t)=(0, t) \in V_{i}^{\prime}=\mathbb{R}^{m-1} \times \mathbb{R}(i=0, \ldots, k-1)$, $f\left(V_{i}^{\prime}\right)^{+} \subset\left(V_{i+1}^{\prime}\right)^{+}, f\left(V_{i}^{\prime}\right)^{-} \subset\left(V_{i+1}^{\prime}\right)^{-}, f\left(\left(V_{i}^{\prime}\right)^{0}\right)=0 \in V_{i+1}^{\prime}=\mathbb{R}^{m}$ for $i=0, \ldots, k-2$,

(3) the restriction of $f$ to $V_{i}^{\prime} \backslash\left(V_{i}^{\prime}\right)^{0}$ is a homeomorphism onto its image $(i=0, \ldots, k-2)$,

(4) there is a Euclidean neighbourhood $W$ such that $V_{0}^{\prime} \subset W, f\left(V_{k-1}^{\prime}\right) \subset W$ and the restriction of $f^{k-1}$ to $W \backslash W^{0}\left(=W^{+} \cup W^{-}=\{(x, t) \in W\right.$; $t \neq 0\})$ is a homeomorphism.

Theorem 3.1 will follow from a sequence of lemmas. Let $f: M \rightarrow M$ satisfy the assumptions of Theorem 3.1 (i.e. of the Cancelling Procedure).

Lemma 3.2. Under the assumptions of the Cancelling Procedure, there is a homotopy $\left\{f_{t}\right\}$ starting from $f_{0}=f$ and an arc $\omega_{0}:[-1,1] \rightarrow M$ from $\omega_{0}(-1)=x_{0}$ to $\omega_{0}(1)=y_{0}$ satisfying:

(1) $\omega_{0}, \omega_{1}=f_{1} \omega_{0}, \ldots, \omega_{S}=f_{1}^{S} \omega_{0}(S=3 k-1)$ are PL-arcs whose interiors, $\omega_{i}(-1,1)$ are mutually disjoint and disjoint from $\operatorname{Fix}\left(f_{1}^{k}\right)$ as indicated in Figure 1,
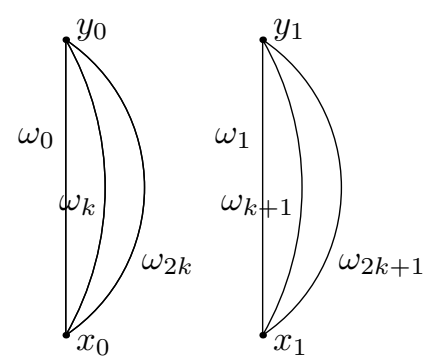

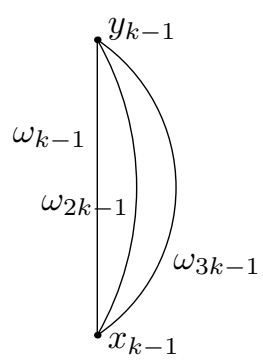

Fig. 1

(2) $\left\{f_{t}\right\}$ is constant in a prescribed neighbourhood of $\operatorname{Fix}\left(f^{k}\right)$,

(3) $\left\{f_{t}\right\}$ can be arbitrarily small,

(4) $\operatorname{Fix}\left(f_{1}^{k}\right)=\operatorname{Fix}\left(f^{k}\right)$.

Proof. We will concentrate on proving (1). Compare Section 3 in [Je].

Since $\operatorname{dim} M \geq 3, \omega_{0}$ may be chosen to be a PL-arc such that $\omega_{0}(t) \notin$ $\operatorname{Fix}\left(f^{k}\right)$ for $-1<t<1$. Since $f$ is a linear homeomorphism in neighbourhoods of $x_{0}$ and $y_{0}$, there exists an $\varepsilon>0$ such that $f \omega_{0}[-1,-1+\varepsilon]$ and 
$f \omega_{0}[1-\varepsilon, 1]$ are segments in the corresponding Euclidean neighbourhoods. Since $\operatorname{dim} M \geq 3$, there exists a small homotopy (rel. ends) on $\omega_{0}[-1+\varepsilon, 1-\varepsilon]$ after which the path $f \omega_{0}[-1+\varepsilon, 1-\varepsilon]$ also becomes a PL-arc; moreover, the homotopy may be extended to a homotopy on the whole $M$ with carrier in a prescribed neighbourhood of $\omega_{0}[-1+\varepsilon, 1-\varepsilon]$. Thus we may assume that $\omega_{1}=f \omega_{0}$ is also an arc and is disjoint from $\omega_{0}[-1,1]$.

We repeat the above construction for the arc $\omega_{1}$ and deduce that $\omega_{2}$ is also an arc disjoint from $\omega_{0}$ and $\omega_{1}$. Thus we may assume that $\omega_{0}, \omega_{1}, \ldots$, $\omega_{k-1}$ are mutually disjoint arcs. Recall that $f$ is a PL-homeomorphism in neighbourhoods of the points $\left\{x_{0}, \ldots, x_{k-1} ; y_{0}, \ldots, y_{k-1}\right\}$. We may continue this procedure to make the $\operatorname{arcs} f^{k}[-1+\varepsilon, 1-\varepsilon], \ldots, f^{S}[-1+\varepsilon, 1-\varepsilon]$ mutually disjoint. Since $f$ is a PL-homeomorphism near the points $\left\{x_{i} ; y_{j}\right\}$ and $\varepsilon>0$ may be arbitrarily small, we may assume that $i \neq j$ and $f^{i} \omega_{0}(t)=f^{j} \omega_{0}(s)$ imply $k \mid(j-i)$ and $t=s= \pm 1$ for $i, j=0, \ldots, S=3 k-1$ (see Fig. 1).

It remains to notice that all the above deformations have carrier isolated from $\operatorname{Fix}\left(f^{k}\right)$. Thus we have (2) and (3), which implies (4).

Set $z_{i}=\omega_{i}(0)$ for $i=0, \ldots, k-1$.

Lemma 3.3. Let $f$ satisfy the assumptions of the Cancelling Procedure. Then there is a homotopy $\left\{f_{t}\right\}$ starting from $f_{0}=f$ satisfying

(1) $\left\{f_{t}\right\}$ is constant in a prescribed neighbourhood of $\operatorname{Fix}\left(f^{k}\right)$,

(2) $\left\{f_{t}\right\}$ can be arbitrarily small,

(3) there exists a neighbourhood $U_{k-1} \ni z_{k-1}$ such that the union

$$
\operatorname{cl} U_{k-1} \cup f_{1}^{-1}\left(\operatorname{cl} U_{k-1}\right) \cup \cdots \cup f_{1}^{-(k-1)}\left(\operatorname{cl} U_{k-1}\right)
$$

is contained in the interior of a finite union of mutually disjoint closed $m$-balls.

Proof. Compare Corollary 4.6 in [Je]. We may assume that $f$ has the properties of $f_{1}$ in Lemma 3.2. Since $\omega_{i}$ are mutually disjoint arcs, we may choose disjoint Euclidean neighbourhoods $V_{i}$ where $\omega_{i}(t)=(0, t) \in V_{i}=$ $\mathbb{R}^{m-1} \times \mathbb{R}(i=0, \ldots, k-1)$. Since the points $z_{i}=\omega_{i}(0)(i=0, \ldots, k-1)$ do not belong to $\operatorname{Fix}\left(f^{k}\right)$, we may deform $f$ in small neighbourhoods $U_{i}$ $\left(z_{i} \in U_{i} \subset V_{i}=\mathbb{R}^{m}\right)$ so that $f$ takes there the form

$$
U_{i} \ni(t, x) \mapsto(t, x) \in V_{i+1} \quad \text { for }(x, t) \in \mathbb{R}^{m-1} \times \mathbb{R} \text { and } i=0, \ldots, k-2 .
$$

Moreover we may assume that $f$ does not vary on $\omega_{i}[-1,1]$ during these deformations. Compare Lemma 4.1 in [Je].

Now we make the map $f$ transverse to the point $z_{k-1}$. The homotopy can be assumed to be arbitrarily small and to be constant in neighbourhoods of the $\operatorname{arcs} \omega_{i}[-1,1]$ for $i=0, \ldots, S-1(=3 k-2)$ since $f^{-1}\left(z_{k-1}\right) \cap \bigcup_{i=0}^{S-1} \omega_{i}[-1,1]=\left\{z_{k-2}\right\}$ and $f$ is a homeomorphism on $U_{k-2} \ni$ 
$z_{k-2}$. Then $f^{-1}\left(z_{k-1}\right)$ becomes a discrete (hence finite) set. Next we notice that $f^{-2}\left(z_{k-1}\right) \cap \bigcup_{i=0}^{S-2} \omega_{i}[-1,1]=\left\{z_{k-3}\right\}$ so we may correct $f$ to obtain a map transverse to $f^{-1}\left(z_{k-1}\right)$ and we may assume as above that the deformation is constant on $\bigcup_{i=0}^{S-3} \omega_{i}[-1,1]$. Continuing this process we get $f, f^{2}, \ldots, f^{k-1}$ transverse to $z_{k-1}$ by means a homotopy constant in a neighbourhood of $\bigcup_{i=0}^{2 k-1} \omega_{i}[-1,1]$ ( since $2 k-1=S-k$ ).

Now $\bigcup_{i=0}^{k-1} f^{-i}\left(z_{k-1}\right)$ is a finite set and $f^{i}$ is a homeomorphism in a neighbourhood of each point of $f^{-i}\left(z_{k-1}\right)(i=1, \ldots, k-1)$. Thus there is an $m$-ball neighbourhood $U^{\prime} \ni z_{k-1}$ such that $W=\bigcup_{i=0}^{k-1} f^{-i}\left(\operatorname{cl} U^{\prime}\right)$ is a finite union of mutually disjoint $m$-balls, each mapped homeomorphically onto $U^{\prime}$. Fix a smaller neighbourhood $U$ satisfying $z_{k-1} \in U \subset \operatorname{cl} U \subset U^{\prime}$. Now $\bigcup_{i=0}^{k-1} f^{-i}(\operatorname{cl} U) \subset W$ (a compact subset of an open set). This proves (3). Recall that the homotopy may be arbitrarily small and constant near $\operatorname{Fix}\left(f^{k}\right)$. Now the other statements of the lemma also follow.

REMARK 3.4. Notice that property (3) of Lemma 3.3 is stable in the following sense: for a given neighbourhood of $\operatorname{Fix}\left(f_{1}^{k}\right)$ there exists a $\delta>0$ such that the conditions $d\left(f_{1}, f^{\prime}\right)<\delta$ and $f_{1}=f^{\prime}$ in this neighbourhood imply that $f^{\prime}$ also satisfies (3) of Lemma 3.3.

LEMMA 3.5. Let $f$ satisfy the assumptions of the Cancelling Procedure. Then there is a homotopy $\left\{f_{t}\right\}$ starting from $f_{0}=f$ and satisfying:

(1) there exist mutually disjoint Euclidean neighbourhoods $V_{0}, \ldots, V_{k-1}$ $\subset M$ where $\omega_{i}(t)=(0, t) \in V_{i}=\mathbb{R}^{m-1} \times \mathbb{R}$ for $-1 \leq t \leq 1$ and $i=0, \ldots, k-1$,

(2) $f_{1}\left(V_{i}^{+}\right) \subset V_{i+1}^{+}, f_{1}\left(V_{i}^{-}\right) \subset V_{i+1}^{-}, f_{1}\left(V_{i}^{0}\right)=z_{i+1} \in V_{i+1}^{0}$ for $i=$ $0, \ldots, k-2$,

(3) the restriction of $f_{1}$ to $V_{i} \backslash V_{i}^{0}$ is a homeomorphism onto its image,

(4) $\left\{f_{t}\right\}$ is constant in a prescribed neighbourhood of $\operatorname{Fix}\left(f^{k}\right)$,

(5) $\left\{f_{t}\right\}$ can be arbitrarily small,

(6) $\operatorname{Fix}\left(f_{t}^{k}\right)$ does not depend on $t$.

Proof. We may assume that $f$ satisfies (3) of Lemma 3.3. We will correct $f$ to make it a homeomorphism near $\omega_{i}[-1,0)$ and $\omega_{i}(0,1]$ for $i=0, \ldots, k-2$. For the details of this construction see Lemma 3.3 and Corollary 4.7 in [Je]. Here we give only an outline of how to make $f$ a homeomorphism near $\omega_{0}[-1,0)$ and $\omega_{0}(0,1]$.

We fix disjoint Euclidean neighbourhoods $U_{0}$ and $U_{1}$ of $\omega_{0}$ and $\omega_{1}=f \omega_{0}$ respectively. We may assume that $\omega_{0}(t)=(0, t) \in \mathbb{R}^{m-1} \times \mathbb{R}=U_{0}$ and $f \omega_{0}(t)=(0, t) \in \mathbb{R}^{m-1} \times \mathbb{R}=U_{1}$.

We fix $m$-simplices $\sigma_{x}, \sigma_{y}$ containing $x_{0}$ and $y_{0}$ respectively on which $f$ is a homeomorphism. Moreover we assume that some $(m-1)$-dimensional faces $\sigma_{-1+\varepsilon} \subset \sigma_{x}, \sigma_{1-\varepsilon} \subset \sigma_{y}$ lie in the hyperplanes $x_{m}=-1+\varepsilon$ and $x_{m}=1-\varepsilon$ 

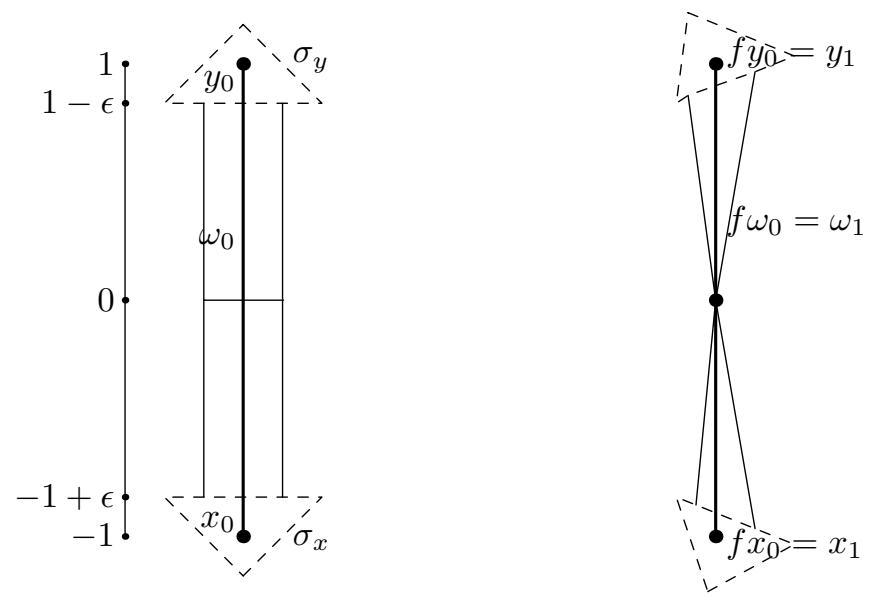

Fig. 2

respectively. Figure 2 illustrates how we will modify $f$ to a homeomorphism near $\omega_{0}[-1,0)$ and $\omega_{0}(0,1]$.

Let $p^{\prime}: \mathbb{R}^{m} \rightarrow \mathbb{R}^{m-1}$ and $p^{\prime \prime}: \mathbb{R}^{m} \rightarrow \mathbb{R}$ denote the projections $p^{\prime}(x, t)=x$ and $p^{\prime \prime}(x, t)=t$ for $(x, t) \in \mathbb{R}^{m-1} \times \mathbb{R}=\mathbb{R}^{m}$. Let $\sigma_{0}$ be an $(m-1)$-simplex contained in $p^{\prime}\left(\sigma_{-1+\varepsilon}\right) \cap p^{\prime}\left(\sigma_{1-\varepsilon}\right)$ and let $0 \in \operatorname{int} \sigma_{0}$. If $\sigma_{0}$ is chosen small enough then $p^{\prime \prime}(f(x,-1+\varepsilon))<0<p^{\prime \prime}(f(x, 1-\varepsilon))$ for $x \in \sigma_{0}$. We define the map $f^{\prime}: \sigma_{0} \times[-1+\varepsilon, 1-\varepsilon] \rightarrow \mathbb{R}^{m}$ by the formula

$$
f^{\prime}(x, t)= \begin{cases}\frac{t}{1-\varepsilon} f(x, 1-\varepsilon) & \text { for } 0 \leq t \leq 1-\varepsilon, \\ \frac{t}{-1+\varepsilon} f(x,-1+\varepsilon) & \text { for }-1+\varepsilon \leq t \leq 0 .\end{cases}
$$

Then the restrictions of $f^{\prime}$ to $\sigma_{0} \times[-1+\varepsilon, 0)$ and to $\sigma_{0} \times(0,1-\varepsilon]$ are homeomorphisms. Fix a number $\eta>0$. If the simplex $\sigma_{0}$ is small enough then $d\left(f^{\prime}(x, t), f(x, t)\right)<\eta$ for $(x, t) \in \sigma_{0} \times[-1+\varepsilon, 1-\varepsilon]$. Moreover the homotopy from $f$ to $f^{\prime}$ (by segments) is still an $\eta$-homotopy and admits an extension onto $M$ which is constant outside a prescribed neighbourhood of $\sigma_{0} \times[-1+\varepsilon, 1-\varepsilon]$. Thus we may assume that after this homotopy no new periodic point (of minimal period $l \leq k, l \mid n)$ appears (Lemma 1.2 in [Je]).

On the other hand $f^{\prime}(x, 1-\varepsilon)=f(x, 1-\varepsilon), f^{\prime}(x,-1+\varepsilon)=f(x,-1+\varepsilon)$ for $x \in \sigma_{0}$ so we may assume that the homotopy is constant on $\sigma_{x}, \sigma_{y}$. For a sufficiently small number $\varepsilon^{\prime}>0$ we have $\sigma_{0} \times\left[1-\varepsilon^{\prime}, 1+\varepsilon^{\prime}\right] \subset \sigma_{y}$ and $\sigma_{0} \times\left[-1-\varepsilon^{\prime},-1+\varepsilon^{\prime}\right] \subset \sigma_{x}$, hence the restriction of $f$ to $\sigma_{0} \times\left(0,1+\varepsilon^{\prime}\right]$ and to $\sigma_{0} \times\left[-1-\varepsilon^{\prime}, 0\right)$ is a homeomorphism. Finally, $V_{0}=\operatorname{int} \sigma_{0} \times\left(-1-\varepsilon^{\prime}, 1+\varepsilon^{\prime}\right)$ is the desired Euclidean neighbourhood.

In general we proceed as follows: we fix a Euclidean neighbourhood $V_{k-1}$ of $\omega_{k-1}$ in which $\omega_{k-1}(t)=(0, t)$. The above construction gives a neighbourhood $V_{k-2} \supset \omega_{k-2}$ such that $f: V_{k-2} \backslash V_{k-2}^{0} \rightarrow V_{k-1}$ is a homeomorphism 
onto its image. Then we choose $V_{k-3}$ and so on. The resulting map satisfies items (1), (2), and (3) of the lemma by the very construction of the homotopy. Since the carrier of the homotopy is contained in (thin) neighbourhoods of the paths $\omega_{i}$ and the homotopy is constant near the ends $x_{i}, y_{i}$ of these paths, (4) holds. Since the above neighbourhoods of the paths $\omega_{i}$ may be arbitrarily thin, (5) is satisfied, which in turn implies (6).

REMARK 3.6. Since the last homotopy may be small, we may assume that the resulting map $f_{1}$ also satisfies Lemma 3.3(3) (see Remark 3.4).

REMARK 3.7. Since $\omega_{k}, \ldots, \omega_{2 k-1}$ are mutually disjoint, so also are $f\left(V_{k-1}\right), \ldots, f^{k}\left(V_{k-1}\right)$ for a sufficiently thin $V_{k-1}$.

The assumption of the next lemma differs from the earlier ones: the path $\omega_{0}$ exceptionally does not join periodic points. This lemma will be applied for $a=1-\varepsilon$ in Remark 3.9 and in the proof of Lemma 3.10.

Lemma 3.8. Let $f: M \rightarrow M$ be a self-map of a compact PL-manifold of dimension $\geq 3$. Assume that

(a) $\operatorname{Fix}\left(f^{k}\right)$ is finite,

(b) $\omega_{0}:[-a, a] \rightarrow M$ is a PL-arc such that $\omega_{i}=f^{i} \omega$ for $i=0, \ldots, 2 k-1$ are mutually disjoint arcs, each disjoint from $\operatorname{Fix}\left(f^{k}\right)$,

(c) $V_{0} \subset M$ is a Euclidean neighbourhood such that $\omega_{0}(t)=(0, t) \in$ $\mathbb{R}^{n-1} \times \mathbb{R}=V_{0}$,

(d) $\omega_{k}(-a), \omega_{k}(a) \in V_{0}, \omega_{k}$ is homotopic (in $M$, rel. end points) to a path lying in $V_{0}$,

(e) there exists a neighbourhood $U_{k-1} \ni z_{k-1}$ such that the union

$$
\operatorname{cl} U_{k-1} \cup f_{1}^{-1}\left(\operatorname{cl} U_{k-1}\right) \cup \cdots \cup f_{1}^{-(k-1)}\left(\operatorname{cl} U_{k-1}\right)
$$

is contained in the interior of a finite union of mutually disjoint m-balls.

Then there is a partial homotopy $h_{s}: \omega_{k-1}[-a, a] \rightarrow M(0 \leq s \leq 2)$ satisfying:

(1) $h_{0}=f_{\mid \omega_{k-1}}, h_{2}$ is a path in $V_{0}, h_{2}\left(\omega_{0}[-a, a]\right) \cap \omega_{0}[-a, a]=\emptyset$,

(2) $h_{s}$ is constant at the ends $\omega_{k-1}(-a)$ and $\omega_{k-1}(a)$,

(3) $f^{i} h_{s} \omega_{k-1}[-a, a]$ is disjoint from $\omega_{k-1}[-a, a]$ for $i=0, \ldots, k-1$,

(4) in particular $f^{i} h_{s}(x) \neq x$ for $x \in \omega_{k-1}[-a, a], 0 \leq s \leq 2, i=$ $0, \ldots, k-1$.

Proof. The partial homotopy $\left\{h_{s}\right\}$ will be obtained in two steps: reparametrization (for $0 \leq t \leq 1$ ) and contraction of the path $f \omega_{k-1}$ to $V_{0}$ (for $1 \leq t \leq 2$ ).

1. Let $\eta>0$ be so small that $0 \times[-\eta, \eta] \subset U_{k-1} \subset \mathbb{R}^{m-1} \times \mathbb{R}=V_{k-1}$ $\left(U_{k-1}\right.$ is the neighbourhood from assumption $\left.(\mathrm{e})\right)$. Let $r:[-a, a] \rightarrow[-a, a]$ 


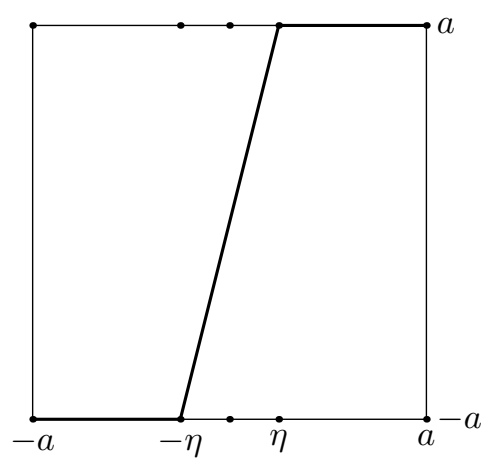

Fig. 3

be the map given by Figure 3, and let $r_{t}: \omega_{k-1}[-a, a] \rightarrow \omega_{k-1}[-a, a]$ be the linear homotopy from $r_{0}=\operatorname{id}_{\omega_{k-1}[-a, a]}$ to $r_{1}\left(\omega_{k-1}(t)\right)=\omega_{k-1}(r(t))$ (reparametrization of the path $\left.\omega_{k-1}\right)$. We define a partial homotopy $h_{t}$ : $\omega_{k-1}[-a, a] \rightarrow M$ putting

$$
h_{t}(x)=f r_{t}(x) \quad \text { for } x \in \omega_{k-1}[-a, a] .
$$

Notice that $r_{1}\left(\omega_{k-1}[-a,-\eta]\right)$ and $r_{1}\left(\omega_{k-1}[\eta, a]\right)$ are points, hence so are $h_{1}\left(\omega_{k-1}[-a,-\eta]\right)$ and $h_{1}\left(\omega_{k-1}[\eta, a]\right)$.

2. The homotopy $h_{s}$ (for $1 \leq s \leq 2$ ) will be constant on $\omega_{k-1}[-a,-\eta]$ and on $\omega_{k-1}[\eta, a]$. We are going to define this homotopy on $\omega_{k-1}[-\eta, \eta]$.

By assumption (d) the restriction of $f$ to $\omega_{k-1}$ is homotopic to a path $\bar{\omega}: \omega_{k-1}[-a, a] \rightarrow V_{0}$, hence also the maps $h_{1}=f r_{1}, \bar{\omega} r_{1}: \omega_{k-1}[-a, a] \rightarrow M$ are homotopic rel. ends. Since both $h_{1}=f r_{1}, \bar{\omega} r_{1}$ are constant on the segments $\omega_{k-1}[-1,-\eta]$ and $\omega_{k-1}[\eta, 1]$, we may assume the same about the homotopy $\left\{h_{s}\right\}(1 \leq s \leq 2)$ between them. We will show that the last homotopy can be made to avoid the set

$$
\operatorname{cl} U_{k-1} \cup f_{1}^{-1}\left(\operatorname{cl} U_{k-1}\right) \cup \cdots \cup f_{1}^{-(k-1)}\left(\operatorname{cl} U_{k-1}\right) .
$$

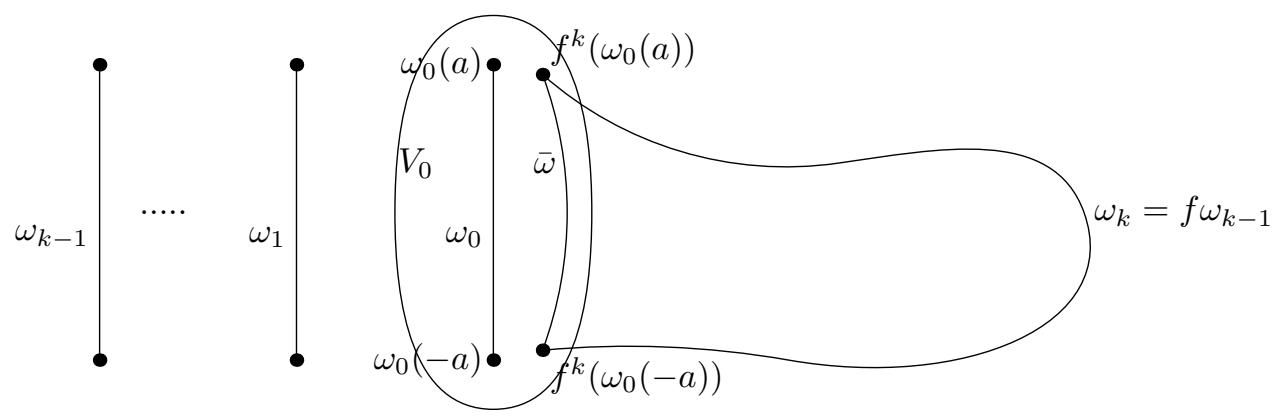

Fig. 4 
In fact by assumption (e) this union is contained in a finite union $\bigcup_{\alpha} K_{\alpha}$ of mutually disjoint balls. Since $\operatorname{dim} M \geq 3$,

$$
\pi_{2}\left(M, M-\bigcup_{\alpha} K_{\alpha}\right)=\pi_{2}(M, M-\text { finite set })=0
$$

so the image of the (two-dimensional homotopy) $h_{s}: \omega_{k-1}[-\eta, \eta] \times[1,2] \rightarrow$ $M$ can be deformed (rel. boundary of $[-\eta, \eta] \times[1,2]$ ) so as to avoid $\bigcup_{\alpha} K_{\alpha}$. Here the assumption $\operatorname{dim} M \geq 3$ is sufficient.

Thus we may assume that $\bar{h}_{s}\left(\omega_{k-1}(t)\right) \notin \bigcup_{i=0}^{k-1} f^{-i}(\operatorname{cl} U)$. Since $\omega_{k-1}[-\eta, \eta]$ $\subset U$ we get $(3)$ and (4) (in $\left.\omega_{k-1}[-\eta, \eta]\right)$. We extend the homotopy $h_{t}$ onto $\omega_{k-1}[-a, a]$ by the constant homotopy outside $\omega_{k-1}[-\eta, \eta]$ and $1 \leq t \leq 2$. Now the homotopy $\left\{h_{t}\right\}$ for $0 \leq t \leq 2$ is as desired.

REMARK 3.9. Let $f=f_{1}: M \rightarrow M$ satisfy (3) of Lemma 3.3 and (1), (2) of Lemma 3.5. Let $\varepsilon>0$ be so small that $f^{k} \omega_{0}[-1,-1+\varepsilon] \cup f^{k} \omega_{0}[1-\varepsilon, 1]$ $\subset V_{0}$. Then the assumptions of Lemma 3.8 are satisfied for $a=1-\varepsilon$.

LEMMA 3.10. The partial homotopy from Lemma 3.8 can be extended to $f_{t}: M \rightarrow M$, where

(1) the carrier of $\left\{f_{t}\right\}$ is contained in $D \times[-1+\varepsilon, 1-\varepsilon] \subset V_{k-1}=$ $\mathbb{R}^{m-1} \times \mathbb{R}$, where $D$ is any prescribed neighbourhood of $0 \in \mathbb{R}^{m-1}$,

(2) $\operatorname{Fix}\left(f_{1}^{k}\right)=\operatorname{Fix}\left(f^{k}\right)$.

Proof. By Remark 3.9 we may assume that we have a partial homotopy $h_{t}: \omega_{k}[-1+\varepsilon, 1-\varepsilon] \rightarrow M$ from Lemma 3.8. Let $D \subset \mathbb{R}^{m-1}$ be a closed ball centred at 0 . Take an arbitrary extension $f_{t}^{\prime}$ of the partial homotopy onto $M$. We consider the metric space $X=M \backslash\left\{\omega_{k-1}(-1+\varepsilon), \omega_{k-1}(1-\varepsilon)\right\}$. Then the sets $X \backslash D \times[-1+\varepsilon, 1-\varepsilon]$ and $0 \times(-1+\varepsilon, 1-\varepsilon)$ are disjoint closed subsets of $X$. Let $\lambda: X \rightarrow[0,1]$ be an Urysohn function satisfying

$$
\lambda|X \backslash D \times[-1+\varepsilon, 1-\varepsilon]=0, \quad \lambda| 0 \times[-1+\varepsilon, 1-\varepsilon]=1 .
$$

Then the map $f_{t}: M \rightarrow M$ defined by

$$
f_{t}(x)= \begin{cases}f_{\lambda(x) t}^{\prime}(x) & \text { for } x \notin\left\{\omega_{k-1}(-1+\varepsilon), \omega_{k-1}(1-\varepsilon)\right\}, \\ f(x) & \text { for } x \in\left\{\omega_{k-1}(-1+\varepsilon), \omega_{k-1}(1-\varepsilon)\right\},\end{cases}
$$

gives a homotopy satisfying $(1)\left(f_{t}\right.$ is continuous at the points $\omega_{k-1}(-1+\varepsilon)$ and $\omega_{k-1}(1-\varepsilon)$ since the homotopy $f_{t}^{\prime}$ is constant there).

It remains to show that if $D$ is small enough then $\operatorname{Fix}\left(f_{2}^{k}\right)=\operatorname{Fix}\left(f^{k}\right)$. Suppose otherwise. Let $D_{n}$ be a concentric ball of radius $1 / n$. Now we have $x_{n} \in D_{n}$ and $0 \leq t_{n} \leq 2$ such that $f_{t_{n}}^{\prime k}\left(x_{n}\right)=x_{n}$. By compactness there are subsequences converging to $x_{0} \in 0 \times[-1+\varepsilon, 1-\varepsilon]$ and $0 \leq t_{0} \leq 2$ respectively. Then $f_{t_{0}}^{k}\left(x_{0}\right)=x_{0}$ contradicts (4) of Lemma 3.8.

REMARK 3.11. Since the only condition on the path $\bar{\omega}$ was $\bar{\omega}(t) \in V_{0} \backslash$ $\omega_{0}[-1,1]$, we may assume $\bar{\omega}(0) \in V_{0}^{-}$. Then $f_{2}\left(z_{k-1}\right)=\bar{\omega}(0) \in V_{0}^{-}$. 
End of the proof of Theorem 3.1. Let $f=f_{1}$ from Lemma 3.10. Then (1)-(3) follow from Lemma 3.5 since Lemma 3.10 does not change $f$ on $V_{0}, \ldots, V_{k-1}$. To get (4) we proceed as follows. We take $W=V_{0}$ and we find $V_{k-1}^{\prime} \subset V_{k-1}$ satisfying the assertion of Lemma 3.5 and $f V_{k-1}^{\prime} \subset W$. If $V_{k-1}^{\prime}$ is thin enough, then $f\left(V_{k-1}^{\prime}\right) \subset W$. Then we find $V_{k-2}^{\prime} \subset V_{k-2}$ satisfying the assertion of Lemma 3.5 and $f V_{k-2}^{\prime} \subset V_{k-1}^{\prime}$ and so on until we get $V_{0}^{\prime} \subset V_{0}$.

4. Extension of the partial homotopy. We will show that the map $f$ satisfying the conclusion ofTheorem 3.1 may be deformed so that $f^{k}$ is given, near the arc $\omega_{0}[-1,1]$, by a prescribed formula. We will need a lemma extending the partial homotopy to a global homotopy with no new periodic points.

We assume that $f: M \rightarrow M$ satisfies the conclusion of Theorem 3.1, and consider the orbits $\left\{x_{0}, \ldots, x_{k-1}\right\},\left\{y_{0}, \ldots, y_{k-1}\right\}$. Since $f$ is a PLhomeomorphism in a neighbourhood of each periodic point and ind $\left(f^{k} ; y_{0}\right)+$ $\operatorname{ind}\left(f^{k} ; x_{0}\right)=0$, we may assume that $\operatorname{ind}\left(f^{k} ; x_{0}\right)=1, \operatorname{ind}\left(f^{k} ; y_{0}\right)=-1$.

In [Je] we considered the map: $h: P \rightarrow \mathbb{R}^{m}\left(P=[-2,2]^{m}\right)$ given by the formula $h(x, t)=\left(\frac{1}{3}|t| x, \eta(t)\right)$ where $(x, t) \in \mathbb{R}^{m-1} \times \mathbb{R}$ and $\eta:[-2,2] \rightarrow \mathbb{R}$ is a function satisfying: $\eta(t)=t \Leftrightarrow t= \pm 1, \eta(-2)>-2, \eta(0)<0, \eta(2)>2$ as indicated in Figure 5.

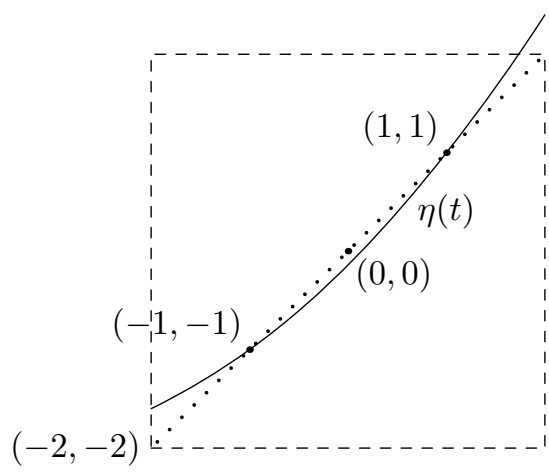

Fig. 5

In this section we will show

THEOREM 4.1. Let the map $f: M \rightarrow M$ satisfy the conclusion of Theorem 3.1. Then there is a homotopy $f_{t}: M \rightarrow M$ with carrier in $V_{k-1}^{\prime}$ satisfying:

(1) $f_{t}\left(V_{k-1}^{\prime}\right) \subset W$

(2) $\operatorname{Fix}\left(f_{t}^{k}\right)$ is constant,

(3) $f_{1}^{k}(x)=h(x)$ for $x \in P=[-2,2]^{k} \subset \mathbb{R}^{m}=V_{0}^{\prime} \subset M$ (here we consider $P \subset \mathbb{R}^{m}$ as a subset of the Euclidean neighbourhood $\left.V_{0}^{\prime}\right)$.

The theorem will follow from a series of lemmas. 
Observe that the restriction $f_{\mid P}^{k}$ and $h: P \rightarrow M$ satisfy

$$
\operatorname{ind}\left(h ; x_{0}\right)=+1=\operatorname{ind}\left(f^{k} ; x_{0}\right), \quad \operatorname{ind}\left(h, y_{0}\right)=-1=\operatorname{ind}\left(f^{k} ; y_{0}\right)
$$

and moreover, if we set $P_{0}=[-2,2]^{m-1} \times 0$, then both $h\left(P_{0}\right)$ and $f^{k}\left(P_{0}\right)$ are points.

The next lemma from [Je] is a modification of the Hopf lemma.

Lemma 4.2. Let $Q=[0,1]^{m}, Q_{0}=[0,1]^{m-1} \times 0$ and let $f_{0}, f_{1}: Q \rightarrow \mathbb{R}^{m}$ be maps satisfying $f_{0}(z) \neq z \neq f_{1}(z)$ for $z \in \operatorname{bd} Q$ and $\operatorname{ind}\left(f_{0}\right)=\operatorname{ind}\left(f_{1}\right)$. Assume moreover that:

(a) $f_{0}\left(Q_{0}\right)$ and $f_{1}\left(Q_{0}\right)$ are points,

(b) $\gamma:[0,1] \rightarrow \mathbb{R}^{m} \backslash Q_{0}$ is a path from $\gamma(0)=f_{0}\left(Q_{0}\right)$ to $\gamma(1)=f_{1}\left(Q_{0}\right)$,

(c) $m \geq 3$.

Then there is a homotopy $H: Q \times I \rightarrow \mathbb{R}^{m}$ satisfying

(1) $H(z, i)=f_{i}(z)$ for $i=0,1, z \in Q$,

(2) $H(z, t) \neq z$ for $t \in I, z \in$ bd $Q$,

(3) $H(z, t)=\gamma(t)$ for $z \in Q_{0} t \in I$.

Proof. See Theorem 5.2 and Lemma 5.3 in [Je].

The above lemma yields a homotopy $\left\{h_{t}\right\}$ from $h_{0}=f^{k}$ (more precisely its restriction to $P$ ) and $h_{1}=h$ (given above by a formula) such that $P_{0}$ is sent to a point and no point from bd $P \cup P_{0}$ is fixed at any moment of this homotopy. This induces a partial homotopy $f_{t}^{\prime}: f^{k-1}(P) \rightarrow M$ by the formula

$$
f_{t}^{\prime}(x)=h_{t}(y) \quad \text { for } x=f^{k-1}(y), y \in P .
$$

In other words, $f_{t}^{\prime}(x)=h_{t}\left(\left(f_{\mid}^{k-1}\right)^{-1}(x)\right)$, where $f_{\mid}^{k-1}$ denotes the restriction $f^{k-1}: P \rightarrow V_{k-1}=\mathbb{R}^{m}$. Since $f_{\mid}^{k-1}$ is injective on $P-P_{0}$ and $h_{t}\left(P_{0}\right)$ is a point (for any fixed $t$ ), the definition is correct. Moreover we recall that the points $f\left(z_{k-1}\right)=f^{k}\left(z_{0}\right)$ and $h\left(z_{0}\right)$ belong to $V_{0}^{-}$(see Remark 3.11 and recall that $h(0,0)=(0, \eta(0))$ where $\eta(0)<0)$. Thus we may join these two points with a path $\gamma:[0,1] \rightarrow V_{0}^{-}$and we may assume that $f_{t}^{\prime}\left(f^{k-1} P_{0}\right)=\gamma(t)$.

Lemma 4.3. The partial homotopy $f_{t}^{\prime}: f^{k-1}(P) \rightarrow M$ admits an extension $f: M \rightarrow M$ with carrier contained in any prescribed neighbourhood of $f^{k-1}(P)$ and such that the set

$$
\left\{x \in \operatorname{Fix}\left(f_{t}^{\prime k}\right) ; \text { the orbit of } x \text { is disjoint from } f^{k-1}(P)\right\}
$$

does not depend on $t$.

Proof. The assertion follows from Lemma 4.4 applied for $X=M, A=$ $f^{k-1}(P), A^{\prime}=U^{\prime}=\emptyset$. It remains only to check that the set

$$
A^{\prime}=\left\{x \in \operatorname{bd} f^{k-1}(P) ; f^{k-1} f_{t}^{\prime}(x)=x \text { for some } x \in f^{k-1}(P)\right\}
$$

is indeed empty. 
Suppose that, on the contrary, $f^{k-1}\left(f_{t}^{\prime}(x)\right)=x$ for some $x=f^{k-1}(y)$, $y \in \operatorname{bd} P$. Assume first that $x=z_{k-1}$. Then $f^{k-1}\left(f_{t}^{\prime}(x)\right)=f^{k-1}\left(h_{t}\left(z_{0}\right)\right)=$ $f^{k-1}(\gamma(t))$. Since $\gamma(t) \in V_{0}^{-}$, we have $f^{k-1}(\gamma(t)) \in V_{k-1}^{-}$, hence $f^{k-1}(\gamma(t))$ $\neq z_{k-1}$.

Now let $x \neq z_{k-1}$. Then $x=f^{k-1}(y)$, where $y \in P \backslash P_{0}$. Since $f_{t}^{\prime}(x)=$ $h_{t}(y)$, the equalities $x=f^{k-1}\left(f_{t}^{\prime}(x)\right)=f^{k-1}\left(h_{t}(y)\right)$ and $x=f^{k-1}(y)$ imply $f^{k-1}\left(h_{t}(y)\right)=f^{k-1}(y)$. Since $f^{k-1}$ is a homeomorphism on $W \backslash W^{0}=$ $W^{+} \cup W^{-}, y=h_{t}(y) \in \operatorname{bd} P$ is a fixed point on the boundary, contradicting the construction of $\left\{h_{t}\right\}$.

Lemma 4.4. Let $A \subset X$ be compact $A N R s, k \in \mathbb{N}, f: X \rightarrow X$ a continuous map, $h_{t}^{\prime}: A \rightarrow X$ a partial homotopy and let $A^{\prime}=\{x \in \operatorname{bd} A ; x=$ $f^{k-1} h_{t}^{\prime}(x)$ for some $\left.t \in[0,1]\right\}$. Moreover, assume that:

(1) $h_{0}^{\prime}(a)=f(a)$ for $a \in A$,

(2) the sets $A, A_{1}=\left\{h_{t}^{\prime}(a) ; a \in A, 0 \leq t \leq 1\right\}, A_{2}=f\left(A_{1}\right), \ldots, A_{k-1}=$ $f^{k-2}\left(A_{1}\right)$ are mutually disjoint,

(3) there exists an open subset $U^{\prime} \subset X$ satisfying:

(a) $h_{t}^{\prime}(x) \in U^{\prime}$ for $x \in A^{\prime}$ and $0 \leq t \leq 1$,

(b) $f^{k-1}\left(\operatorname{cl} U^{\prime}\right) \subset A$.

Then there exists an extension of the partial homotopy $\left\{h_{t}^{\prime}\right\}$ to $h_{t}: X \rightarrow X$ satisfying:

(1) $h_{0}=f$

(2) the carrier of the homotopy $\left\{h_{t}\right\}$ is contained in an arbitrarily prescribed neighbourhood of $A$,

(3) $\left\{x \in \operatorname{Fix}\left(h_{t}^{k}\right)\right.$; the orbit of $x$ is disjoint from $\left.A\right\}$ does not depend on $t \in[0,1]$.

Proof. Let $H: X \rightarrow X$ be an arbitrary extension of the partial homotopy $h_{t}^{\prime}$ to $X$. Then $H\left(A^{\prime} \times I\right) \subset U^{\prime}$, hence by compactness there exists an open subset $U \subset X$ containing $A^{\prime}$ and satisfying $H(\operatorname{cl} U \times I) \subset U^{\prime}$. Moreover if $U$ is sufficiently small then, by assumption (2), the sets

$$
\operatorname{cl} U \cup A, B_{1}=H((\operatorname{cl} U \cup A) \times I), B_{2}=f\left(B_{1}\right), \ldots, B_{k-1}=f^{k-2}\left(B_{1}\right)
$$

are mutually disjoint. Then setting $h_{t}^{\prime}=H(\cdot, t)$ we have $\left(h_{t}^{\prime}\right)^{k}(x)=f^{k-1} h_{t}^{\prime}(x)$ for all $x \in \operatorname{cl} U$.

We will show that $\left(h_{t}^{\prime}\right)^{k}(x) \neq x$ for all $x \in \operatorname{cl} U \backslash A$ and $t \in[0,1]$. In fact $x \in \operatorname{cl} U \backslash A$ implies $x \in \operatorname{cl} U$, hence $h_{t}^{\prime}(x) \in U^{\prime}$. Now $\left(h_{t}^{\prime}\right)^{k}(x)=f^{k-1} h_{t}^{\prime}(x) \in$ $f^{k-1} U^{\prime} \subset A$ implies $\left(h_{t}^{\prime}\right)^{k}(x) \neq x$ since $x \notin A$.

Now we show that $\left(h_{t}^{\prime}\right)^{k}(x) \neq x$ for all $x \in \operatorname{bd}(A \cup \operatorname{cl} U)$. Suppose otherwise, i.e. $\left(h_{t}^{\prime}\right)^{k}(x)=x$. We notice that $\operatorname{bd}(A \cup \operatorname{cl} U) \subset \operatorname{bd} A \cup \operatorname{bd}(\operatorname{cl} U)$. First we assume that $x \in \operatorname{bd} A$. Then the equality $\left(h_{t}^{\prime}\right)^{k}(x)=x$ implies $x \in A^{\prime}$ so $x \in A^{\prime} \subset U \subset \operatorname{int}(A \cup \operatorname{cl} U)$ and hence $x \notin \operatorname{bd}(A \cup \operatorname{cl} U)$, contrary to the 
assumption. If $x \in \operatorname{bd}(\operatorname{cl} U) \backslash \operatorname{bd} A$ then $x \in \operatorname{cl} U \backslash A$ and $\left(h_{t}^{\prime}\right)^{k}(x) \neq x$ as above.

Let $B=\left\{x \in X \backslash A ;\left(h_{t}^{\prime}\right)^{k}(x)=x\right.$ for some $\left.t \in I\right\}$. By the above, $B$ is a closed subset of $X$ disjoint from $A$. Let $V$ be a neighbourhood of $A$. Let $\lambda: X \rightarrow[0,1]$ be an Urysohn function with $\lambda \mid A=1$ and $\lambda \mid B \cup(X \backslash V)=0$. We will show that $h_{t}(x)=h_{\lambda(x) t}^{\prime}(x)$ is the desired homotopy. In fact (1) is evident. To see that $(2)$ holds we notice that $h_{t}(x)=h_{\lambda(x) t}^{\prime}(x)=h_{0}^{\prime}(x)=$ $f(x)$ for all $x \notin V$. It remains to show (3). Fix $t \in[0,1]$ and consider an orbit of $h_{t}$ avoiding $A$. This orbit must lie in $B$. Now $h_{t}(x)=h_{\lambda(x) t}^{\prime}(x)=$ $h_{0}^{\prime}(x)=f(x)$ for any $x$ from the orbit, which implies that this is also an orbit of $f$. The same arguments show that each orbit of $f=h_{0}$ avoiding $A$ lies in $B$ and is an orbit of $h_{t}$, which gives (3).

Proof of Theorem 4.1. As we have noticed the modified Hopf lemma induces a partial homotopy $f_{t}^{\prime}$ (on $f^{k-1}(P)$ ) starting from $f$ and so that $f_{1}^{\prime} f^{k-1}=h$ on $P \subset \mathbb{R}^{m}=V_{0}^{\prime}$. Then Lemma 4.3 extends the local homotopy to a global one. The carrier of $f_{t}$ may be as small as we please, hence we can assume that it is contained in $V_{k-1}^{\prime}$. On the other hand, since $f^{k-1} f_{1}^{\prime}(x)=x$ iff $x=x_{k-1}$ or $x=y_{k-1}$, the condition on orbits in Lemma 4.3 makes $\operatorname{Fix}\left(f_{t}^{k}\right)$ independent of $t$.

\section{End of the proof of the Cancelling Procedure (Theorem 2.5).} We may assume that $f: M \rightarrow M$ satisfies the conclusion of Theorem 3.1 and by the last section we may moreover assume that $f^{k}(x, t)=\left(\frac{1}{3}|t| x, \eta(t)\right)$ for $(x, t) \in P \subset W=\mathbb{R}^{m}$.

We will use the following technical lemma (proved in [Je]).

Lemma 5.1 ([Je, Lemma 5.5]). There is a homotopy $h_{s}: P \rightarrow \mathbb{R}^{m}$ $(0 \leq s \leq 3)$ satisfying

(1) $h_{0}(x, t)=\left(\frac{1}{3}|t| x, \eta(t)\right)$,

(2) $h_{s}\left(P_{0}\right)$ is a point, for each fixed $s$,

(3) $h_{s}(z) \neq z$ for $z \in$ bd $P$,

(4) $h_{s}(x, t)=h_{0}(x, t)$ for $(x, t) \in \operatorname{bd} P,|t| \geq \varepsilon$,

(5) $h_{s}(x, t) \in \operatorname{int} P$ for $|t|<\varepsilon$,

(6) $h_{3}(z) \neq z$ for all $z \in P$,

(7) $h_{3}(0) \in \operatorname{int} P \backslash P_{0}$.

Since $f^{k-1}$ is a homeomorphism on $P \backslash P_{0}$, the homotopy $h_{s}$ from Lemma 5.1 induces a partial homotopy $h_{s}^{\prime}: f^{k-1}(P) \rightarrow M$ by the formula $h_{s}^{\prime}(x)=$ $h_{s}\left(\left(f_{\mid}^{k-1}\right)^{-1}(x)\right)$. The definition is correct by property (2) because $f^{k-1}$ is a homeomorphism on $P \backslash P_{0} \subset W \backslash W^{0}$.

We will show 
Claim 1. Lemma 4.4 can be applied to the above partial homotopy with $X=M, \quad A=f^{k-1}(P), \quad A^{\prime}=f^{k-1}\left(P_{0}\right)=z_{k-1}=$ a point.

This gives a homotopy $\left\{\tilde{h}_{s}\right\}, 0 \leq s \leq 3$, starting from $\tilde{h}_{0}=f$.

Claim 2. $\operatorname{Fix}\left(\tilde{h}_{3}^{k}\right)=\operatorname{Fix}\left(f^{k}\right) \backslash\left\{x_{0}, \ldots, x_{k-1} ; y_{0}, \ldots, y_{k-1}\right\}$.

Now $\tilde{h}_{3}$ is the desired map homotopic to $f$ satisfying the Cancelling Procedure.

It remains to prove the above two claims.

Proof of Claim 1. We show that the assumptions of Lemma 4.4 are satisfied.

(1) is evident.

(2) We recall that the sets $W, f(W), \ldots, f^{k-2}(W)$ are disjoint from $f^{k-1}(W)$ (if only $W$ was chosen sufficiently thin-see Remark 3.7). On the other hand, $A_{1}=\bigcup_{t} h_{t}^{\prime}\left(f^{k-1} P\right)=\bigcup_{t} h_{t}(P) \subset \mathbb{R}^{m}=W$ hence $A_{2} \subset$ $f(W), \ldots, A_{k-1} \subset f^{k-2}(W)$ are disjoint from $A=f^{k-1}(P) \subset f^{k-1}(W)$.

(3) We begin by showing that $f^{k-1} h_{t}^{\prime}(v) \neq v$ for any $v \in \operatorname{bd} f^{k-1}(P) \backslash$ $z_{k-1}$. Let $v \in \operatorname{bd}\left(f^{k-1}(P)\right)$. Then $v=f^{k-1}(z)$ for some $z \in \operatorname{bd} P$. Suppose that $f^{k-1} h_{t}^{\prime}(v)=v$. Then $f^{k-1} h_{t}^{\prime}(v)=f^{k-1}(z)$. Suppose that $z \notin P_{0}$. Since the restriction of $f^{k-1}$ to $P \backslash P_{0}$ is injective, we have $h_{t}^{\prime}(v)=z$. Thus $z=h_{t}^{\prime}(v)=h_{t}^{\prime} f^{k-1}(z)=h_{t}(z)$, contrary to Lemma 5.1(3).

Now we prove that assumptions (a) and (b) are satisfied.

Let $U^{\prime}=$ int $P$. By Lemma 5.1(5), $h_{s}\left(P_{0}\right) \subset U^{\prime}$, hence $h_{s}^{\prime}\left(z_{k-1}\right)=$ $h_{s}\left(P_{0}\right) \subset U^{\prime}$ and so (a) is satisfied. On the other hand, the equality $f^{k-1}\left(\mathrm{cl} U^{\prime}\right)$ $=f^{k-1}(P)=A$ proves $(\mathrm{b})$.

Proof of Claim 2. We will show that $\tilde{h}_{3}^{k}(z) \neq z$ for all $z \in f^{k-1}(P)$. Suppose otherwise. Thus $z \in \operatorname{Fix}\left(\tilde{h}_{3}^{k}\right) \cap f^{k-1}(P)$ implies $\tilde{h}_{3}(z) \in \operatorname{Fix}\left(\tilde{h}_{3}^{k}\right)$. Then the point $y=\tilde{h}_{3}(z)$ belongs to $P \subset W$. In fact, since $z=\tilde{h}_{3}^{k-1}(y)=$ $f^{k-1}(y), z \in f^{k-1}(P)$ and $f^{k-1}$ is injective on $W^{+} \cup W^{-}$, it follows that $y \in P$. Now $\tilde{h}_{3}^{k}(y)=h_{3}^{\prime}\left(f^{k-1}(y)\right)=h_{3}(y)$. On the other hand, Lemma $5.1(6)$ implies $h_{3}(y) \neq y$. This contradicts $z \in \operatorname{Fix}\left(\tilde{h}_{3}^{k}\right)$.

The above, Lemma 4.4 and the equality $\tilde{h}_{3}=f$ imply

$$
\begin{aligned}
\operatorname{Fix}\left(\tilde{h}_{3}^{k}\right) & =\left\{x \in \operatorname{Fix}\left(\tilde{h}_{3}^{k}\right) ; \text { the orbit of } x \text { is disjoint from } f^{k-1} P\right\} \\
& =\left\{x \in \operatorname{Fix}\left(\tilde{h}_{0}^{k}\right) ; \text { the orbit of } x \text { is disjoint from } f^{k-1} P\right\} \\
& =\operatorname{Fix}\left(f^{k}\right) \backslash\left\{x_{0}, \ldots, x_{k-1} ; y_{0}, \ldots, y_{k-1}\right\} .
\end{aligned}
$$

\section{References}

[Je] J. Jezierski, Cancelling periodic points, Math. Ann. 321 (2001), 107-130.

[Ji1] B. J. Jiang, Lectures on the Nielsen Fixed Point Theory, Contemp. Math. 14, Amer. Math. Soc., Providence 1983. 
[Ji2] B. J. Jiang, Fixed point classes from a differential viewpoint, in: Lecture Notes in Math. 886, Springer, 1981, 163-170.

[K] M. Kelly, Minimizing the number of fixed points for self-maps of compact surfaces, Pacific J. Math. 126 (1987), 81-123.

[W] F. Wecken, Fixpunktklassen, I-III, Math. Ann. 117 (1941), 659-671; 118 (1942), 216-234; 118 (1942), 544-577.

Institute of Mathematics

University of Agriculture

Nowoursynowska 159

02-787 Warszawa, Poland

E-mail: jezierski@alpha.sggw.waw.pl

Received 17 February 2003;

in revised form 2 October 2003 and 29 January 2004 